\title{
Impacto do Programa de Aquisição de Alimentos na Segurança Alimentar e Nutricional dos agricultores
}

\author{
Impact of the Food Acquisition Program on the food \\ and nutrition security of the farmers
}

Silmara Christina Rodrigues de Assis ${ }^{1}$

Silvia Eloíza Priore ${ }^{2}$

Sylvia do Carmo Castro Franceschini ${ }^{2}$

${ }^{1}$ Programa de Pós-

Graduação em

Agroecologia. Universidade

Federal de Viçosa. Av.

P.H. Rolfs s/n, Campus

Universitário. 36570-

900 Viçosa MG Brasil.

silmaranutri@

yahoo.com.br

${ }^{2}$ Departamento de Nutrição

e Saúde, UFV. Viçosa MG

Brasil.

\begin{abstract}
The scope of this systematic review was to analyze the impact of the Food Acquisition Program on the diversification of production, onfarm consumption and food and nutritional security of the family-based farmer and supplier beneficiaries. Searches were conducted in the Lilacs and SciELO electronic databases and the Capes journal portal. The publications were pre-selected based on their titles, followed by reading of the abstracts and the full text of the studies. Articles that evaluated the program's implementation, along with those that referred to food and nutrition security of consumers who benefitted from the program, as well as repeat and review articles, were excluded. Of the 193 studies reviewed, 7 were chosen. In all of those selected, there were positive impacts of the Food Acquisition Program on increasing family income, benefiting from access to the market and moving their produce. This made it possible to improve the production system and fostered crop diversification both for trade and for on-farm consumption of the families, having a positive impact on the food and nutrition security situation of the farmers.
\end{abstract}

Key words Food and nutrition programs and policies, Food and nutrition security, Sustainable agriculture, Rural population
Resumo Objetivou-se nesta revisão sistemática analisar o impacto do Programa de Aquisição de Alimentos na diversificação da produção, autoconsumo e segurança alimentar e nutricional dos agricultores familiares beneficiários fornecedores. Realizou-se consulta nas bases de dados eletrônicas Lilacs, SciELO e no portal de periódicos da Capes. As publicações foram pré-selecionadas pelos títulos, acompanhada da leitura dos resumos e na integra dos estudos, se excluiu aqueles que avaliavam a execução do Programa, que se referiam à segurança alimentar e nutricional dos beneficiários consumidores, artigos repetidos e de revisão. Dos 193 estudos revisados selecionou-se 7. Observou-se em todos os selecionados impactos positivos do Programa de Aquisição de Alimentos no aumento da renda familiar, favorecida pela garantia de acesso ao mercado e o escoamento da produção, viabilizando melhorias no sistema de produção e incentivando o cultivo diversificado tanto para comercialização quanto para autoconsumo da família, inferindo positivamente na situação de Segurança Alimentar e Nutricional dos agricultores. Palavras-chave Programas e políticas de nutrição e alimentação, Segurança alimentar e nutricional, Agricultura sustentável, População rural 


\section{Introdução}

No meio rural a pobreza e a fome são resultados, em grande parte, da exclusão dos pequenos produtores, pois durante muitos anos as políticas públicas para o espaço rural privilegiaram a agricultura patronal, direcionadas para as médias e grandes propriedades, visando à produção para exportação e focalizada, sobretudo, a produção em grande escala ${ }^{1,2}$.

A agricultura familiar foi, por muito tempo, tida como agricultura de subsistência. Atualmente é indiscutível a sua importância na produção agrícola, por ser a principal fornecedora de produtos considerados básicos para alimentação humana ${ }^{3}$ e cumprir um papel decisivo na produção de alimentos para o mercado interno ${ }^{4}$. Segundo dados do Ministério do Desenvolvimento Agrário $2015^{5}$, na agricultura familiar domina a produção de mandioca (87\%), feijão (70\%), carne suína (59\%), leite (58\%), carne de aves (50\%) e milho (46\%).

O termo agricultura familiar surgiu no Brasil durante a década de $1990^{6-8}$, período marcado por uma efervescência dos movimentos sociais do campo ligados aos pequenos produtores e ao movimento dos Trabalhadores sem Terra ${ }^{3,8,9}$, diante dos desafios que o sindicalismo rural enfrentava nesta época - impactos da abertura comercial, falta de crédito agrícola e queda dos preços dos principais produtos agrícolas de exportação ${ }^{10}$.

Questões como participação social, segurança alimentar e desenvolvimento local também ganhavam espaço crescente na concepção das políticas de desenvolvimento rural, cenário onde a agricultura familiar é peça fundamental. Assim, estratégias de desenvolvimento local sustentável e de geração de novas oportunidades de trabalho começam a ser reivindicadas pelos agricultores ${ }^{8}$.

Foi nesse contexto, que se concebeu em 1996, com o intuito de fornecer crédito e assistência técnica, o Programa Nacional de Fortalecimento da Agricultura Familiar (PRONAF), um marco de afirmação da importância do pequeno produtor familiar, que foi por muitos anos menos beneficiados pelas políticas públicas agrícolas ${ }^{11}$. A partir de 2000, os movimentos sociais da agricultura familiar passaram a reivindicar ações que ultrapassavam o escopo de atividades exercidas pelo PRONAF, exigindo apoio à comercialização dos seus produtos, acesso aos mercados e garantia de preços ${ }^{11}$, fatores que consistiam em um dos maiores entraves para o desenvolvimento econômico do segmento ${ }^{8}$.
Diante das novas reivindicações por parte dos agricultores familiares, aliado a uma preocupação do governo federal frente à população em situação de fome, miséria e insegurança alimentar ${ }^{12}$, em 2003 é criado Programa de Aquisição de Alimentos (PAA), dentro de um grupo de políticas estruturantes da Estratégia Fome Zero, visando implementar ações no âmbito das políticas agrícolas e de segurança alimentar e nutricional $^{13}$. Sua execução é voltada para melhorias quanto ao acesso aos alimentos das pessoas em vulnerabilidade social e alimentar, por meio da compra governamental de produtos da agricultura familiar e distribuição para entidades sociassistenciais $^{14}$.

Segundo Sambuichi et al. ${ }^{15}$ as avaliações do PAA em 29 estudos, indicam um programa que tem atuado na alteração da matriz produtiva dos agricultores familiares, o impacto mais comum foi a diversificação da produção, relatada em 72\% dos estudos, seguida por melhorias na qualidade dos alimentos produzidos, fortalecimento das organizações coletivas e aumento da renda familiar. Esses três impactos foram relatados em 52\% dos estudos.

Para Maluf ${ }^{16}$, o PAA tem o papel primordial de romper com o círculo vicioso da fome, e enfrentar a pobreza rural que é um dos principais focos de insegurança alimentar. Os resultados da Pesquisa Nacional por Amostra de Domicílios de $2009^{17}$ revelaram que a prevalência de famílias em situação de insegurança alimentar na zona rural $(35,1 \%)$ foi maior que na zona urbana $(29,4 \%)$. Gazolla $^{18}$ afirma que a "pobreza e a situação de insegurança alimentar na zona rural surgem com a especialização produtiva, onde os agricultores familiares se destinam aos monocultivos, visando renda, obtendo pouca produção para autoconsumo e adquirindo alimentos em mercados locais".

O Relatório de Insegurança Alimentar no Mundo de $2014^{19}$, publicado pela FAO, revelou que o Brasil reduziu de forma muito expressiva a fome, a desnutrição e a subalimentação nos últimos anos. Os avanços decorrem, na análise apresentada, da priorização da agenda de Segurança Alimentar e da implementação, de forma articulada, de programas de proteção social e de fomento à produção agrícola, como o PAA ${ }^{19,20}$.

Neste contexto, avaliar o impacto de um programa é de fundamental importância, pois enseja apontar prováveis mudanças ocorridas na vida dos beneficiários, e averiguar se os objetivos do mesmo têm sido efetivados, propiciando resultados satisfatórios ${ }^{21}$. No caso do PAA, que é caracterizado por um programa que, em virtude do 
seu conjunto de ações, relaciona a produção com o consumo, os beneficiários podem ser classificados em dois grupos: os fornecedores que são os agricultores familiares que vendem seus produtos para o programa e os consumidores que são todos os grupos de pessoas beneficiadas por esses alimentos ${ }^{22}$. Apesar da grande e crescente quantidade de pesquisas envolvendo o PAA, poucos trabalhos visam avaliar de forma detalhada o impacto do programa para produtores familiares beneficiários ${ }^{21}$.

Diante do exposto, o presente artigo de revisão sistemática objetiva analisar o impacto do Programa de Aquisição de Alimentos na diversificação da produção, autoconsumo e Segurança Alimentar e Nutricional dos agricultores familiares beneficiários fornecedores.

\section{Metodologia}

Trata-se de uma revisão sistemática de literatura construída no período de outubro a dezembro de 2014, na Universidade Federal de Viçosa, Minas Gerais. A revisão foi realizada centrada na pergunta norteadora: "O Programa de Aquisição de Alimentos é capaz de contribuir com a segurança alimentar e nutricional dos agricultores familiares?”.

Como estratégia de busca para seleção dos estudos foram consultadas a base de dados Literatura Latino Americana e do Caribe em Ciências da Saúde (Lilacs), Scientific Electronic Library Online (SciELO) e o portal de periódicos da Capes. A busca nas fontes supracitadas foi realizada tendo como termos indexadores Programas e Políticas de Nutrição e Alimentação Programa de Aquisição de Alimentos, Segurança Alimentar e Nutricional, Segurança Alimentar Agricultura Sustentável, Agricultor Familiar, Autoconsumo e diversificação da produção de alimentos, a pesquisa foi realizada combinando-se esses termos ou utilizando-os de forma isolada.

Realizou-se busca reversa, pesquisando as listas de referência dos estudos, com a finalidade de identificar artigos originais publicados e não localizados. Consultou-se também leis, decretos, entre outros, documentos governamentais do Ministério do Desenvolvimento Social e Combate a Fome, Ministério de Desenvolvimento Agrário, Ministério da Agricultura, Pecuária e Abastecimento, Conselho Nacional de Segurança Alimentar e Nutricional e Companhia Nacional de Abastecimento.

Para a revisão, considerou-se o período desde 2003, ano de promulgação da Lei 10.696, de 2 de julho, art. $19^{23}$ que configura o PAA como instrumento de política pública, até o ano de 2014. Inicialmente, as publicações foram pré-selecionadas pelos títulos, os quais deveriam conter como primeiro critério o termo completo e/ou referências ao menos um dos desfechos de interesse, acompanhada da leitura dos resumos disponíveis e posteriormente a leitura na integra dos estudos. Após a leitura total dos estudos incluiu-se nesta revisão sistemática artigos originais, de cunho nacional, que apresentavam associação entre a política de compras do PAA com SAN dos agricultores familiares beneficiários fornecedores. Não foram incluídos estudos que não contemplavam os objetivos desta revisão, foram excluídos artigos de revisão, estudo que avaliavam a implantação e execução do PAA e os artigos que avaliaram o impacto do PAA na SAN dos beneficiários consumidores.

A busca eletrônica em base de dados associada à busca reversa e a pesquisa nos portais de instituições públicas resultou na identificação inicial de 193 trabalhos. Após o primeiro refinamento, selecionou-se 30, cujos títulos ou resumos mencionavam as contribuições do PAA à SAN de agricultores familiares beneficiários fornecedores. No segundo refinamento, selecionou-se 7 publicações (5 artigos, 1 documento do Departamento de Estudos Socioeconômicos Rurais e 1 dissertação de mestrado). Excluiu-se 186 estudos que não contemplavam os objetivos dessa revisão, artigos repetidos e aqueles que não apresentaram dados originais para a avaliação de possíveis impactos do PAA na segurança alimentar dos agricultores familiares beneficiários fornecedores (Figura 1).

\section{Resultados}

O Quadro 1 apresenta uma síntese dos dados principais dos artigos em estudo. Quanto ao público alvo, os trabalhos de Mattei ${ }^{13}$, Becker e Sacco dos Anjos ${ }^{24}$, Silva ${ }^{25}$, Hespanhol ${ }^{26}$ e, Silva e Almei$\mathrm{da}^{27}$ tiveram como público alvo os agricultores familiares beneficiados pelo PAA, as pesquisas do Departamento de Estudos Sócio-Econômicos Rurais (DESER) ${ }^{28}$ e de Silva e Almeida ${ }^{27}$, também incorporaram à amostra representantes da CO$\mathrm{NAB}$, secretaria de agricultura, movimento dos trabalhadores rurais e do Conselho Estadual de Segurança Alimentar, e, sindicatos de agricultores, respectivamente. O estudo de Santos et al. ${ }^{14}$ realizou-se com agentes técnicos de extensão rural. 


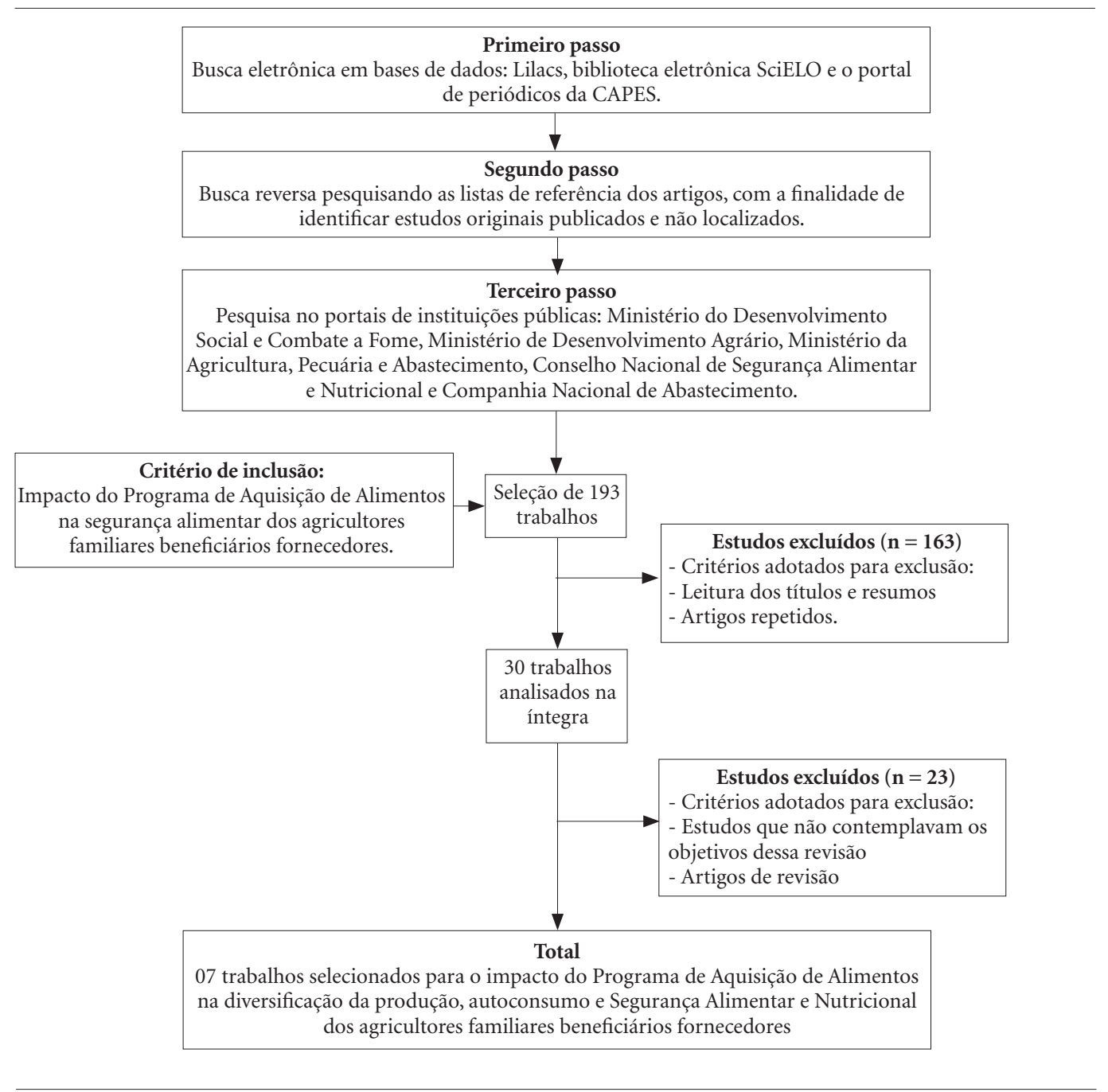

Figura 1. Protocolo de busca nas bases de dados científicas e portais de organizações nacionais e critério de inclusão dos estudos para análise.

Verificou-se, que o PAA contribuiu para a consolidação do mercado local, segurança de comercialização e fortalecimento da agricultura familiar ${ }^{28}$, propiciando, de maneira comum, importante impacto sobre o aumento da renda monetária mensal das familias ${ }^{13,14,24-28}$ e evitando o êxodo rural ${ }^{25}$. O DESER observou, inclusive, que o programa encorajou os produtores a investirem em capacitação profissional e reestruturação dos sistemas de produção, adequando-os às práticas agroecológicas ou aquelas que utilizavam em menor grau insumos químicos ${ }^{28}$. Outras melhorias efetivadas foram ampliação da área cultivada, aumento da produção e da produtividade, percebido nos estudos de Mattei ${ }^{13}$, Santos et al. ${ }^{14}$, DESER ${ }^{28}$ e Hespanhol ${ }^{26}$. Mattei ${ }^{13}$ destacou ainda o progresso tecnológico na unidade produtiva, Silva ${ }^{25}$ apontou a especialização para o trabalho na agroindustrialização, já $\mathrm{DESER}^{28}$, Becker e Sacco dos Anjos ${ }^{24}$ enfatizaram o resgate e a valorização de práticas agrícolas e produtos tradicionais.

Ainda sobre esta perspectiva, Silva e Almei$\mathrm{da}^{27}$, com objetivo de avaliar a experiência do PAA, por intermédio da modalidade Compra com Doação Simultânea para a aquisição de sementes crioulas, no Polo da Borborema, visualizou um conjunto de resultados importantes para o fortalecimento da estratégia de produção e comercialização, entre eles a retomada de estra- 
Quadro 1. Levantamento de estudos realizado sobre Programa de Aquisição de Alimentos e o seu impacto sobre a Segurança Alimentar e Nutricional dos agricultores familiares beneficiários fornecedores.

\begin{tabular}{|c|c|c|c|c|}
\hline $\begin{array}{l}\text { Autor/ } \\
\text { Ano }\end{array}$ & $\begin{array}{l}\text { Local de } \\
\text { estudo }\end{array}$ & Metodologia & $\begin{array}{c}\text { Característica da } \\
\text { população }\end{array}$ & Resultados \\
\hline $\begin{array}{l}\text { Mattei } \\
(2007)^{13}\end{array}$ & $\begin{array}{l}\text { Estado: Santa } \\
\text { Catarina } \\
\text { Municípios: } \\
\text { São Miguel } \\
\text { do Oeste, } \\
\text { Guaraciaba } \\
\text { e Dionísio } \\
\text { Cerqueira }\end{array}$ & $\begin{array}{l}\text { Delineamento: } \\
\text { Estudo de caso. } \\
\text { Aplicação de } \\
\text { questionários. }\end{array}$ & $\begin{array}{l}61 \text { agricultores familiares } \\
\text { beneficiários } \\
7 \text { associações de } \\
\text { agricultores }\end{array}$ & $\begin{array}{l}\text { - Aumento da renda } \\
\text { familiar dos agricultores. } \\
\text { - Estímulo à produção de } \\
\text { novos produtos. } \\
\text { - Melhorias tecnológicas } \\
\text { no sistema produtivo. } \\
\text { - Ampliação da área de } \\
\text { produção }\end{array}$ \\
\hline $\begin{array}{l}\text { Deser } \\
(2008)^{28}\end{array}$ & $\begin{array}{l}\text { Região } \\
\text { Nordeste } \\
\text { Estados: Bahia, } \\
\text { Pernambuco e } \\
\text { Ceará }\end{array}$ & $\begin{array}{l}\text { Delineamento: } \\
\text { Estudo de caso. } \\
\text { Entrevista com } \\
\text { vários atores } \\
\text { envolvidos no } \\
\text { PAA. }\end{array}$ & $\begin{array}{l}\text { Representantes da: } \\
\text { - Companhia Nacional de } \\
\text { Abastecimento } \\
\text { - Secretaria Estadual } \\
\text { da Agricultura e do } \\
\text { Desenvolvimento Social } \\
\text { - Conselho Estadual de } \\
\text { Segurança Alimentar } \\
\text { - Confederação Nacional } \\
\text { dos Trabalhadores na } \\
\text { Agricultura } \\
\text { - Federação dos } \\
\text { Trabalhadores na } \\
\text { Agricultura Familiar do } \\
\text { Brasil. } \\
\text { - Movimento dos } \\
\text { Trabalhadores Rurais. } \\
\text { - Agricultores familiares. }\end{array}$ & $\begin{array}{l}\text { - Aumento da produção. } \\
\text { - Diversificação da } \\
\text { produção. } \\
\text { - Melhoria dos alimentos } \\
\text { para comercialização e } \\
\text { autoconsumo familiar. } \\
\text { - Incentivo as práticas } \\
\text { agroecológicas. } \\
\text { - Resgate e valorização de } \\
\text { produtos tradicionais. } \\
\text { - Capacitação } \\
\text { profissional. } \\
\text { - Segurança da } \\
\text { comercialização. } \\
\text { - Aumento da renda } \\
\text { familiar. } \\
\text { - Melhoria da qualidade } \\
\text { de vida. }\end{array}$ \\
\hline
\end{tabular}

continua

tégias tradicionais de cultivo como os consórcios que diversifica a alimentação e os produtos a serem comercializados.

Em todos os estudos foi determinante o papel do PAA na diversificação produtiva, pois o programa abarca vários grupos de alimentos, tanto in natura quanto processados, o que também influenciou positivamente na manutenção ou mesmo o incremento das práticas de autoconsumo, promovendo a SAN do grupo familiar, visto que consumiam uma variedade de alimentos produzidos por eles mesmos, observando os hábitos locais e reproduzindo um cenário relevante para a qualidade de vida no meio rural ${ }^{13,14,24-28}$.

Diante do exposto, relatos de agricultores familiares apresentados no estudo de Becker e Sac- co dos Anjos ${ }^{24}$ nos auxiliam a compreender esta situação: Antes quando queria se comer bem, se ia ao restaurante na cidade. Ali se dizia que se comia bem. Agora eu acho que aqui em casa a gente tem a mesma diversidade do restaurante. Não perdemos em nada pros restaurantes e com a vantagem de ser um produto de qualidade, sem veneno e adubo químico. Tudo saudável e produzido na propriedade ${ }^{24}$.

Se come mais porque se tem mais produtos disponíveis. As verduras ajudam a ter mais opções. Vai da lavoura para a mesa ${ }^{24}$.

A gente tomava remédio pra pressão... agora com uma alimentação saudável e o trabalho que a gente faz, não precisamos mais de remédio. Se alimentando bem, a saúde melhora ${ }^{24}$. 
Quadro 1. continuação

\begin{tabular}{|c|c|c|c|c|}
\hline $\begin{array}{l}\text { Autor/ } \\
\text { Ano }\end{array}$ & $\begin{array}{l}\text { Local de } \\
\text { estudo }\end{array}$ & Metodologia & $\begin{array}{l}\text { Característica da } \\
\text { população }\end{array}$ & Resultados \\
\hline $\begin{array}{l}\text { Becker } \\
\text { e Sacco } \\
\text { dos Anjos } \\
(2010)^{24}\end{array}$ & $\begin{array}{l}\text { Estado: Rio } \\
\text { Grande do Sul } \\
\text { Município } \\
\text { Pelotas, } \\
\text { Canguçu e São } \\
\text { Lourenço do } \\
\text { Sul. }\end{array}$ & $\begin{array}{l}\text { Delineamento: } \\
\text { Estudo de caso. } \\
\text { Questionários } \\
\text { e entrevista, } \\
\text { composta por } \\
\text { perguntas } \\
\text { fechadas e } \\
\text { abertas. }\end{array}$ & $\begin{array}{l}60 \text { famílias rurais } \\
\text { beneficiárias }\end{array}$ & $\begin{array}{l}\text { - Aumento da renda familiar. } \\
\text { - Resgate de atividades } \\
\text { agrícolas tradicionais. } \\
\text { - Diversificação produtiva. } \\
\text { - Diversificação produtiva } \\
\text { interferiu positivamente } \\
\text { na Segurança Alimentar e } \\
\text { Nutricional dos agricultores } \\
\text { familiares. }\end{array}$ \\
\hline $\begin{array}{l}\text { Santos et } \\
\text { al. }(2012)^{14}\end{array}$ & $\begin{array}{l}\text { Estados: Minas } \\
\text { Gerais e Bahia }\end{array}$ & $\begin{array}{l}\text { Delineamento: } \\
\text { Estudo de caso. } \\
\text { Aplicação de } \\
\text { questionários. }\end{array}$ & $\begin{array}{l}449 \text { Agentes Técnicos } \\
\text { de } \\
\text { Extensão Rural dos } \\
\text { Estados de Minas } \\
\text { Gerais e Bahia }\end{array}$ & $\begin{array}{l}\text { - Ampliação da renda } \\
\text { familiar. } \\
\text { - Aumento do volume de } \\
\text { produção e diversificação da } \\
\text { produção de alimentos. } \\
\text { - Fortalecimento da } \\
\text { agricultura familiar. } \\
\text { - Diminuiu a insegurança } \\
\text { alimentar }\end{array}$ \\
\hline $\begin{array}{l}\text { Silva } \\
(2013)^{25}\end{array}$ & $\begin{array}{l}\text { Estado: Paraná } \\
\text { Município: } \\
\text { Copanema }\end{array}$ & $\begin{array}{l}\text { Delineamento: } \\
\text { Estudo de caso. } \\
\text { Entrevista } \\
\text { semiestruturada } \\
\text { constituída de } \\
\text { questões abertas } \\
\text { e fechadas. }\end{array}$ & $\begin{array}{l}12 \text { agricultores } \\
\text { familiares associados à } \\
\text { cooperativa. }\end{array}$ & $\begin{array}{l}\text { - Aumento da renda familiar. } \\
\text { - Diversificação da produção. } \\
\text { - Especialização para } \\
\text { agroindustrialização. } \\
\text { - Melhoria na qualidade } \\
\text { de vida da família tanto na } \\
\text { questão financeira, quanto } \\
\text { na questão social, de saúde } \\
\text { e na Segurança Alimentar e } \\
\text { Nutricional. }\end{array}$ \\
\hline
\end{tabular}

continua

\section{Discussão}

Apesar de inúmeras pesquisas envolvendo o PAA, poucas visam avaliar o impacto do programa para SAN dos produtores beneficiados, a maioria dos trabalhos se debruça a estudar os agricultores enquanto fornecedores de alimentos que abastecem o mercado, fortalecendo sua relação comercial e obtendo ganhos sociais e econômicos. A contribuição do PAA para promoção da SAN predomina nas pesquisas com foco nas entidades receptoras dos alimentos e nos beneficiários consumidores.

Para avaliação da situação de alimentação e nutrição de populações em vulnerabilidade social não há um único indicador considerado padrão ouro, mas é possível obter combinação de indicadores que avaliem diferentes aspectos como indicadores socioeconômicos, de saúde, comportamento e a percepção dos indivíduos estudados ${ }^{29}$. Os métodos comumente utilizados para mensurar a insegurança alimentar, Escala Brasileira de Insegurança Alimentar (EBIA), antropometria e investigação do consumo alimentar individual ${ }^{30,31}$, não constavam na metodologia dos estudos que avaliaram o impacto do PAA na SAN dos agricultores.

A SAN foi avaliada em todos os estudos por questionários semiestruturados não validados para população e elaborados pelos autores, que fizeram alusão à melhoria da renda advinda da adesão ao PAA, à melhor qualidade de vida, ao estímulo à produção diversificada para venda $\mathrm{e}$ ao autoconsumo inferindo positivamente na situação de SAN dos agricultores e de sua família. 
Quadro 1. continuação

\begin{tabular}{|c|c|c|c|c|}
\hline Autor/Ano & Local de estudo & Metodologia & $\begin{array}{c}\text { Característica da } \\
\text { população }\end{array}$ & Resultados \\
\hline $\begin{array}{l}\text { Hespanhol } \\
(2013)^{26}\end{array}$ & $\begin{array}{l}\text { Estado: São Paulo } \\
\text { Município: } \\
\text { Dracena }\end{array}$ & $\begin{array}{l}\text { Delineamento: } \\
\text { Estudo de caso. } \\
\text { Entrevistas. }\end{array}$ & $\begin{array}{l}60 \text { produtores } \\
\text { beneficiários. }\end{array}$ & $\begin{array}{l}\text { - Melhoria da renda familiar.. } \\
\text { - Incentivo a diversificação } \\
\text { produtiva. } \\
\text { - Aumento da produção } \\
\text { de alimentos para atender } \\
\text { ao consumo familiar e } \\
\text { as necessidades da rede } \\
\text { socioassistencial local e } \\
\text { regional. } \\
\text { - Promoção e valorização dos } \\
\text { hábitos alimentares locais. }\end{array}$ \\
\hline $\begin{array}{l}\text { Silva e } \\
\text { Almeida } \\
(2013)^{27}\end{array}$ & $\begin{array}{l}\text { Estado: Paraíba } \\
\text { Polo da Borborema }\end{array}$ & $\begin{array}{l}\text { Delineamento: } \\
\text { Estudo caso. } \\
\text { Entrevistas } \\
\text { semiestruturadas }\end{array}$ & $\begin{array}{l}15 \text { sindicatos de } \\
\text { trabalhadores } \\
\text { rurais envolvendo } \\
16 \text { municípios e } \\
\text { agricultores. }\end{array}$ & $\begin{array}{l}\text { - Resgate da técnica de } \\
\text { plantio em consórcio } \\
\text { Diversificação dos sistemas } \\
\text { produtivos. } \\
\text { - Diversificação da } \\
\text { alimentação da família do } \\
\text { agricultor. } \\
\text { - Aumento da renda familiar. }\end{array}$ \\
\hline
\end{tabular}

Sabe-se que indicadores socioeconômicos são uma das formas de mensurar a insegurança alimentar já que domicílios com acesso precário a renda, bens e serviços estão mais vulneráveis a risco de insegurança alimentar ou fome e às repercussões da mesma, como alterações nutricionais ${ }^{32,33}$. Ressalta-se que um indicador, isolado, não consegue abranger as múltiplas dimensões de SAN, uma vez que cada método monitora o fenômeno, segundo ótica própria, tornando os demais complementares ${ }^{30}$.

De acordo com Conselho Nacional de Segurança Alimentar e Nutricional ${ }^{34}$ e Garret e Ruel ${ }^{35}$, o conceito de SAN abrange disponibilidade, acesso e suficiência alimentar. A disponibilidade e o acesso são os fatores que estão mais diretamente associados à renda familiar e às características do mercado de alimentos da região, que envolvem as possibilidades de produção e os sistemas de preço e de logística. Além disso, há também a possibilidade de as famílias contarem com produção própria ou doação de alimentos. Já a suficiência alimentar é uma medida mais complexa, que depende da articulação entre disponibilidade, acesso e distribuição do consumo intrafamiliar, ou seja, a suficiência é influenciada pelo tamanho e pela composição das famílias, assim como por escolaridade, cultura e renda ${ }^{34,35}$.

Diante do exposto, pode-se dizer que os estudos primários que compuseram a presente revisão avaliaram o impacto do PAA na situação da SAN dos agricultores apenas sob a ótica da disponibilidade e acesso aos alimentos.

Os estudos revelaram o programa como instrumento para melhorar a situação alimentar das famílias em vulnerabilidade. Nesse grupo estão inseridos além dos consumidores beneficiários do programa, os produtores. De acordo com Bragatto $^{36}$, quanto ao agricultor, a melhoria da situação alimentar pode ocorrer da seguinte forma: com a aquisição de alimentos da agricultura familiar pelo PAA o governo apoia a produção para o autoconsumo e adquire o excedente por um preço de referência, propicia a renda e possibilita o acesso aos alimentos em quantidade e qualidade, garantindo a SAN.

Em todos os estudos foi unânime a referência aos impactos positivos do PAA sobre o aumento da renda familiar, favorecida pela garantia de acesso ao mercado e o escoamento da produção, viabilizando melhorias no sistema de produção e incentivando o cultivo diversificado tanto para 
comercialização quanto para autoconsumo da família, refletindo positivamente na situação de SAN dos agricultores.

Para Grisa et al. ${ }^{37}$ os agricultores familiares que possuem o autoconsumo diversificado nas suas unidades de produção, provavelmente irão gastar menos com a compra de alimentos, podendo utilizar os recursos economizados em outras necessidades essenciais. Ademais, produzindo para autoconsumo na unidade de produção, os agricultores não se tornam vulneráveis em relação à oferta e aos preços dos alimentos nos mercados.

Além disto, a qualidade nutricional dos alimentos para autoconsumo é sempre interpretada como geradora da segurança alimentar por geralmente conter qualidade nutritiva que seria "superior" aos alimentos comprados. Isto se deve ao fato de que, geralmente, esta prática é realizada sem agrotóxicos e outros produtos químicos ${ }^{37}$.

A importância da produção para o autoconsumo não representa apenas o alimento, mas a cultura, os costumes de uma família ou comunidade. Neste sentido, Menasche et al..$^{38}$ apontam que a produção de alimentos para o autoconsumo tende [...] a assegurar a segurança alimentar, uma vez que, estando enraizada na história vivida pelas famílias e pela comunidade, tem por atributos a diversidade, a quantidade e a disponibilidade $d u$ rante todo o ano ${ }^{38}$.

A agricultura familiar condiciona todas as possibilidades de alimentar os membros do grupo doméstico com alimentação diversificada e que contenha todos os tipos de alimentos ${ }^{33}$, cumprindo o princípio da alimentação saudável, que é a utilização de todos os grupos alimentares na composição da dieta diária. A diversidade da dieta que fundamenta o conceito de alimentação saudável pressupõe que nenhum alimento específico ou grupo deles isoladamente é suficiente para fornecer todos os nutrientes necessários a uma boa nutrição e consequente manutenção da saúde, é nesse sentido que a autoprodução diversificada de alimentos se insere ${ }^{39,40}$.

É importante ressaltar que a diversificação da produção para consumo da família no meio rural estimulada pelo PAA pode ser um caminho para se reverter ou diminuir o resultado encontrado pela Pesquisa de Orçamento Familiar (POF) entre os anos de 2008 à 2009, em uma avaliação sobre à quantidade de alimentos consumidos pela família na zona rural, 45,6\% das famílias referiram algum grau (normalmente e eventualmente) de insuficiência da quantidade de alimentos consumido ${ }^{41}$.

Contudo, nos estudos analisados o delineamento foi do tipo transversal, havendo limitações que impedem inferências mais abrangentes. Nesse sentido, os resultados não permitem o estabelecimento de relações causais.

Outro aspecto limitante diz respeito à metodologia dos artigos primários incluídos nesta revisão, a população de estudo abarcou vários informantes, produtores, agentes técnicos de extensão rural, representantes de sindicados de agricultores e movimentos dos trabalhadores rurais, secretária de agricultura e conselho de segurança alimentar, de maneira não sistematizada, o que pode ter interferido nos resultados, visto que, por se tratarem de trabalhos que buscavam discutir os impactos do PAA para os agricultores beneficiados, somente estes são aptos a revelar as mudanças ocorridas a partir da adesão ao programa, os outros atores envolvidos no processo poderiam ser incluídos visando complementar a investigação e não como informante chave.

\section{Conclusões}

Como mostrou este artigo, o PAA tem propiciado aos agricultores beneficiados a possibilidade de se inserir no mercado local, contribuindo para aumentar a receita monetária da família, além de estimular os produtores a incorporarem um modelo diversificado de produção de alimentos, assegurando uma variedade necessária tanto para abastecer o mercado quanto para o autoconsumo, inferindo positivamente na situação de SAN dos agricultores e de sua família.

A temática abordada nesta revisão almeja aguçar a discussão sobre o tema, para que futuros estudos utilizem protocolos e padrões de avaliação que garantam qualidade metodológica para avaliação da SAN dos agricultores beneficiados pelo PAA, com indicadores definidos e mensurados de forma adequada. Finalmente, pretende-se contribuir com uma referência científica a fim de auxiliar na ampliação das ações do PAA voltadas a mitigar a pobreza, a fome e a situação de insegurança alimentar ainda presente no meio rural. 


\section{Colaboradores}

A autora SCR Assis trabalhou na concepção, redação final e revisão e as autoras SE Priore e SCC Franceschini na redação final e revisão crítica.

\section{Referências}

1. Rocha AGP, Cerqueira PS, Coelho VP. Um panorama do Programa de Aquisição de Alimentos no estado da Bahia: estudos de caso em Boa Vista do Tupim, Tapiramutá e Vitória da Conquista. Rev Sociedade e Desenvolvimento Rural 2007; 1(1):1-22

2. Vieira DFA. Influência do Programa de Aquisição de Alimentos na comercialização dos produtos da agricultura familiar: o caso do município de Paracatu em Minas Gerais [dissertação]. Brasília: Universidade de Brasília; 2008.

3. Brandão AA. Produção e Comercialização de Hortaliças em Feiras Livres na microrregião de Januária [dissertação]. Montes Claros: Universidade Federal de Minas Gerais; 2012.

4. Câmara Interministerial de Segurança Alimentar e Nutricional (Caisan). Plano nacional de segurança alimentar e nutricional - 2012-2015. Brasília: Caisan; 2011. [acessado 2015 mar 5]. Disponível em: http://www. mds.gov.br/segurancaalimentar/publicacoes\%20sisan/ livros/plano-nacional-de-seguranca-alimentar-e-nutricional-2012-2015/.

5. Brasil. Ministério do Desenvolvimento Agrário (MDA). A força da agricultura familiar. Brasília: MDA; 2015. [acessado 2015 ago 21]. Disponível em: http:// www.mda.gov.br/sitemda/noticias/for $\%$ C $3 \%$ A7a-da -agricultura-familiar.

6. Denardi RA. Agricultura familiar e politicas públicas: alguns dilemas e desafios para o desenvolvimento rural sustentável. Rev Agroecol e Desenv Rur Sustent 2001; 2(3):56-62.

7. Schneider S. Teoria Social, agricultura familiar e pluriatividade. Rev Bras Ci Soc 2003; 18(51):99-123.

8. Cerqueira OS, Rocha AGP, Coelho VP. Agricultura familiar e políticas públicas: algumas reflexões sobre o Programa de Aquisição de Alimentos no estado da Bahia. Rev Desenbahia 2006; 3(5):55-78.

9. Centro Internacional de Políticas para o Crescimento Inclusivo Programa das Nações Unidas para o Desenvolvimento (IPC-IG). Demanda Estruturada e Pequenos Agricultores no Brasil: o Caso do PAA e PNAE. Brasília: 2013.

10. Schneider S. Agricultura familiar e desenvolvimento rural endógeno: elementos teóricos e um estudo de caso. In: Froehlich JM, Vivien D, organizadores. Desenvolvimento Rural - Tendências e debates comtemporâneos. Ijuí: Unijuí; 2006. p. 1-24.

11. Muller AL. A construção das políticas públicas para a Agricultura Familiar no Brasil: o caso do Programa de Aquisição de Alimentos [dissertação]. Porto Alegre: Universidade Federal do Rio Grande do Sul; 2007.

12. Gazolla M, Schneider S. O papel da agricultura familiar para a segurança alimentar: uma análise a partir do programa Fome Zero no município de Constantina/RS. Sociedade e Desenvolvimento Rural 2007; 1(1):85-102.

13. Mattei L. Programa de Aquisição de Alimentos da Agricultura Familiar (PAA):antecedentes, concepção e composição geral do programa. Cadernos do CEAM (UnB) 2007; 7(1):33-44.

14. Santos AR, Vieira NS, Ferreira PR, Castro TTS. Agricultura familiar e Segurança Alimentar e Nutricional: análise dos resultados do programa de aquisição de alimentos (PAA doação simultânea) nos estados da Bahia e Minas Gerais. Cadernos Gestão Social 2012; 3(1):9-24. 
15. Sambuichi RHR, Galindo EP, Oliveira MAC, Moura AMM. Compras públicas sustentáveis e agricultura familiar: a experiência do Programa de Aquisição de Alimentos (PAA) e do Programa Nacional de Alimentação Escolar (PNAE). In: Sambuichi RHR, Silva APM, Oliveira MAC, Savia A, organizadores. Políticas agroambientais e sustentabilidade: desafios, oportunidades e lições aprendidas. Brasília: IPEA; 2014. p. 75-104.

16. Maluf RS. Políticas agrícolas e de Desenvolvimento rural e de segurança alimentar. In: Leite S, organizador. Politicas Públicas e Agricultura no Brasil. Porto Alegre: Editora da UFRGS; 2001. p. 58-85.

17. Instituto Brasileiro de Geografia e Estatística (IBGE). Pesquisa Nacional por Amostra de Domicílios - PNAD - Despesas, rendimento e Condições de Vida: 2008/2009. Rio de Janeiro: IBGE; 2010.

18. Gazolla M. Agricultura familiar, segurança alimentar e políticas publicas: uma análise a partir da produção para autoconsumo no território do Alto Uruguai/RS [dissertação]. Porto Alegre: Universidade Federal do Rio Grande do Sul; 2004.

19. Food and Agriculture Organization of the United Nations (FAO). The State of Food Insecurity in the World 2014. Strengthening the enabling environment for food security and nutrition. Rome: FAO; 2014.

20. Food and Agriculture Organization of the United Nations (FAO). O estado da Segurança Alimentar e Nutricional no Brasil: um retrato multidimensional. Relatório 2014. Brasília: FAO; 2014.

21. Rossi FR. Programa de Aquisição de Alimentos (PAA) da agricultura familiar no município de São Carlos:impactos socioeconômicos nos agricultores familiares beneficiários [dissertação]. São Carlos: Universidade Federal de São Carlos; 2012.

22. Guerra JS. O Programa de Aquisição de Alimentos no Âmbito do Programa Fome Zero: emancipação ou compensação? [dissertação]. Pelotas: Universidade de Pelotas; 2010.

23. Brasil. Lei no 10.696, de 2 de julho de 2003. Dispõe sobre a repactuação e o alongamento de dívidas oriundas de operações de crédito rural, e dá outras providências. Diário Oficial da União 2003; 3 jul.

24. Becker C, Sacco dos Anjos F. Segurança Alimentar e desenvolvimento rural: limites e possibilidades do Programa de Aquisição de Alimentos da agricultura familiar, em municípios do Sul Gaúcho. Rev Segurança Alimentar e Nutricional 2010; 17(1):61-72.

25. Silva V. O Papel do Programa de Aquisição de alimentos - PAA - para o fortalecimento da agricultura familiar: $o$ caso da Cooperativa da Agricultura Familiar Rural Integrada - COOPAFI - de Capanema, PR [dissertação]. Pato Branco: Universidade Tecnológica Federal do Paraná; 2013.

26. Hespanhol RAM. Programa de Aquisição de Alimentos: limites e potencialidades de políticas de Segurança Alimentar para a agricultura familiar. Rev Soc Nat 2013; 25(3):469-483.

27. Silva ED, Almeida MF. Programa de Aquisição de Alimentos: tecendo os caminhos entre segurança alimentar e a política de sementes no semiárido paraibano. Cadernos de Agroecologia 2013; 8(2):1-6.
28. Departamento de Estudos Sócio-Econômicos Rurais (Deser). O Programa de Aquisição de Alimentos e sua relação com a Política Nacional de Segurança Alimentar e Nutricional e a Política de Comercialização Agrícola no Brasil, entre 2003-07: Uma Avaliação. Curitiba: Deser; 2008.

29. Corrêa AMS. Insegurança alimentar medida a partir da percepção das pessoas. Estud Av 2007; 21(60):143-154.

30. Pérez-Escamilla R. Seguridad Alimentaria Y Nutricional: Marco Conceptual. In: XII Congresso Brasileiro de Sociologia, 2005, Belo Horizonte. Sociologia e realidade: pesquisa social no século XXI, 2005.

31. Segall-Corrêa AM. Insegurança alimentar medida a partir da percepção das pessoas. Estud Av 2007; 21(60):143-154.

32. Salles-Costa R, Pereira RA, Vasconcellos MTL, Veiga GV, Marins, VMR, Jardim BC, Gomes FS, Sichieri R. Associação entre fatores socioeconômicos e insegurança alimentar: estudo de base populacional na Região Metropolitana do Rio de Janeiro, Brasil. Rev Nutr 2008; 21(Supl.):99-109.

33. Campbell CC. Food insecurity: a nutritionaloutcome or a predictor variable? J Nutr 1991; 121(3):408-415.

34. Conselho Nacional de Segurança Alimentar e Nutricional (Consea). A Segurança Alimentar e Nutricional e o direito à alimentação adequada no Brasil. Indicadores e monitoramento, da Constituição de 1988 aos dias atuais. Brasília: Consea; 2010.

35. Garret JL, Ruel MT. Are determinants of rural and urban food security and nutritional status different? Some insights from Mozambique. Washington: Food Policy Research Institute; 1999. [acessado 2015 jan 25]. Disponível em: http://www.ifpri.org/sites/default/files/ pubs/divs/fcnd/dp/papers/dp65.pdf

36. Bragatto SA. Programa de Aquisição de Alimentos: segurança alimentar e inclusão socialcno campo?[tese]. São Paulo: Universidade de São Paulo; 2010.

37. Grisa C, Gazolla M, Schneider S. A "produção invisível" na agricultura familiar: autoconsumo, Segurança Alimentar e políticas públicas de desenvolvimento rural. Rev Agroalimentaria 2010; 16(31):65-79.

38. Menasche R, Marques FC, Zanetti C. Autoconsumo e segurança alimentar: a agricultura familiar a partir dos saberes e práticas da alimentação. Rev Nutr 2008; 21(Supl.):145-158.

39. Deves OD. Fortalecimento da agricultura familiar através do Programa de Aquisição de Alimentos - PAA : o caso do município de São Pedro do Butiá-RS [dissertação]. Porto Alegre: Universidade Federal do Rio Grande do Sul; 2009.

40. Bezerra I, Schneider S. Produção e consumo de alimentos: o papel das políticas públicas na relação entre o plantar e o comer. Rev. UFRGS 2012; 15(20):36-61.

41. Instituto Brasileiro de Geografia e Estatística (IBGE). Pesquisa de Orçamentos Familiares 2008/2009. Rio de Janeiro: IBGE; 2010.

Artigo apresentado em 11/05/2015

Aprovado em 02/10/2015

Versão final apresentada em 04/10/2015 